\title{
Electromagnetic Field Analysis and Shielding Method of Underground Variable Frequency Power Cable
}

\author{
Jianwei Zhang (D) and Zhuojing Yang \\ Center for Hydrogeology and Environmental Geology, China Geological Survey, Baoding 071051, Hebei, China \\ Correspondence should be addressed to Jianwei Zhang; chegs_jianwei@st.btbu.edu.cn
}

Received 29 October 2021; Accepted 6 December 2021; Published 5 January 2022

Academic Editor: Sang-Bing Tsai

Copyright (C) 2022 Jianwei Zhang and Zhuojing Yang. This is an open access article distributed under the Creative Commons Attribution License, which permits unrestricted use, distribution, and reproduction in any medium, provided the original work is properly cited.

\begin{abstract}
The transmission and radiation of underground variable frequency electromagnetic waves will seriously interfere with the operation of the power cable and its surrounding environment. At present, the test methods for power cables basically require the impedance of the test system to match the characteristic impedance of the cable. The defect is that the process of designing and making the impedance matching impedance network is relatively complex and requires high manufacturing accuracy. In order to solve these problems, this paper puts forward the electromagnetic field fast detection formula and electromagnetic field shielding method of underground variable frequency power cable. The research method of this paper is the principle of shielding electromagnetic field materials and the suppression principle of shielding layer for electromagnetic coupling. The function of the two principles is to study the reflection, absorption, and multiple reflection of electromagnetic waves and to study the cut-off frequency of the nonmagnetic shielding layer. These two principles guide the experiment. In this paper, the measurement formula of the shielding performance of mismatched cables is derived through experiments. The results show that the error of the measurement formula is no more than $8 \mathrm{~dB}$. Then, through the experiment of restraining the interference of magnetic materials on the electromagnetic field, it is concluded that the magnetic field shielding performance can reach $20 \mathrm{~dB}$. Then, through the performance test of electromagnetic field shielding materials, the shielding efficiency of metal fiber antiradiation materials is the largest, and the average efficiency reaches $76.4 \mathrm{~dB}$.
\end{abstract}

\section{Introduction}

1.1. Background. The power cable is mainly used as the feeder of wireless transmitting and receiving equipment and is used as the internal connection line and wiring of various communication equipment and electronic equipment. They are widely used in microwave relay, radar, communication, radio, television, military, aerospace, and other fields. They are an important communication part of the device. The cable itself is an effective receiving and radiating antenna. There is a large distributed capacity and mutual inductance between conductors, resulting in signal leakage between conductors. When a conductor passes through an electric current, there is an electric and magnetic field around it. If the electromagnetic field reaches a certain strength, it may have a bad impact on the surrounding metal parts and electronic machines. In order to eliminate its influence, various measures have been taken to protect the electromagnetic field. The shielding effect of the shielding component is caused by the absorption attenuation and reflection attenuation of electromagnetic waves. Absorption attenuation is the main shielding mode of low-frequency electromagnetic waves, and reflection attenuation is the main shielding mode of high-frequency electromagnetic waves. In the United States, experts in electromagnetic field research also point out that more and more people are increasing the incidence rate of cancer and other diseases due to long-term exposure to electromagnetic radiation, and the high-frequency electromagnetic waves are more harmful to the basic tissues of organisms and have long-term and potential hazards to human health. It will cause strong interference and even disastrous consequences to all kinds of civil or military electronic and electrical products [1]. 
1.2. Significance. No matter in life or in the operation of underground power cables, human beings must shield and protect as much as possible to prevent the harm of electromagnetic radiation. Therefore, the research on electromagnetic rapid measurement technology and the development of shielding materials has certain practical significance and far-reaching impact. Electromagnetic shielding has become an indispensable part of cable operators. Through experiments, the factors affecting the shielding efficiency of electromagnetic shielding materials are found out, which provides a theoretical basis for the production of such materials in the future.

1.3. Related Work. There has been a lot of research on solving the problem of underground power cable electromagnetic fields. Li et al. developed a dynamic model of the underground electromechanical system, proposed a realtime voltage and frequency optimization scheme, and simulated the real-time frequency optimization scheme and constant frequency real-time voltage regulation scheme based on the principle of constant voltage-frequency ratio, in order to reduce load fluctuation and reduce the required motor rating and energy consumption. The results show that the real-time frequency optimization scheme can significantly reduce the rated value of the motor, while the constant frequency real-time voltage regulation scheme can provide a significant energy-saving effect. The real-time frequency optimization scheme combines the advantages of the two control methods and reduces the variation range of voltage, so as to improve the service life of the motor. Although constant frequency has many advantages, however, its operational flexibility and emergency response capacity will be greatly reduced [2]. Tian et al. believe that, in complex downhole operations, with the increase of drilling depth, there are many new problems or challenges such as low ROP, serious stick slip, and high risk of downhole tool failure, and the working conditions of drilling engineering become more and more complex. The key factor to solve these new challenges is to improve rock-breaking efficiency. He proposed a new oscillator based on a positive displacement motor, designed a new structure, and, combined with new complex drilling conditions, proposed a mathematical model, including the newly designed working mechanism and structural parameters. Although the vibration displacement, system vibration spectrum, and velocity at different drill string positions can be obtained in this way, he ignores the interference of electromagnetic field on downhole operation, and it is not enough to only solve the rock-breaking efficiency [3]. Samuel proposed that the inflow and migration of fluid in the wellbore have a great impact on the damping coefficient, so it is necessary to monitor the high-frequency acceleration data for early detection. When gas, fluid, or oil surge occurs, fluid inflow will reduce the viscosity of the fluid in the annulus, reduce the damping coefficient, and endanger the normal use of power cable. He proposed the deconvolution method of sensor value, which uses minimum entropy deconvolution and energy operator to remove noise and unwanted sensor value.
However, when considering various adverse factors, he ignored the electromagnetic field caused by the cable itself, leaving hidden dangers [4]. Rajpoot et al. propose a SUGPDS model based on detection and isolation algorithm and intelligent sensor, which is used to identify, classify, and isolate various faults, supervise, manage and control various faults and problems. When the underground distribution system fails, the model can maintain the uninterrupted flow, stability, and reliability of power. Although this method plays an important role in identifying and classifying faults, quickly isolating faults from healthy networks, and helping to reduce power consumption, it is too dependent on the installation location of intelligent sensing and switchgear [5]. Famakin and Kim believe that underground cable faults, whether temporary or permanent, can be traced back to insulation faults, most of which are caused by electromagnetic fields. Insulation damage usually leads to costly power cuts, which can be prevented by taking preemptive actions. The most effective action is to track the magnetic field in the cable insulation system in real time and model the magnetic field. However, the models he uses are static, because they focus on the changes of cable insulation characteristics in a certain period of time. Therefore, a new electromagnetic field modeling method is needed, focusing on the parabolic form changes of model values [6]. Hadi believes that economic constraints and lack of sufficient space will require cables with higher current carrying capacity. Attention should be paid to improving the accuracy level within the range of cable safety parameter values. Accurate parameter values are very important to ensure safety. This process involves making magnetic field and temperature field distribution models check the magnetic field and temperature distribution in the cable area and deploying cables. This work conceives an innovative process for calculating cable thermal field and current carrying capacity using the finite element method, but the use of igital paths may be more specific and flexible [7]. In terms of shielding electromagnetic field, Lovat et al. believe that, in addition to the parallel admittance related to shielding conductivity, the existence of a magnetic field will also introduce the series impedance into the equivalent network of the structure. In addition, the series impedance will produce an integral similar to that generated by shunt admittance. Therefore, he proposed a generalized analysis formula for combining magnetic and conductive screens, compared with the classical approximate formula and accurate results; it is more advantageous in downhole operation. However, the series impedance and electromagnetic field do not interact, so they can be handled independently [8]. Xiaodong et al. successfully prepared layered cotton material with excellent conductivity and electromagnetic shielding performance by grafting graphene and then electroless plating $\mathrm{Ni}$. This layer-by-layer structure design fully reflects the synergistic effect of wave reflection and wave absorption. They also tested the mechanical properties in practical use and passed a series of tests; they believe that the composite has good application potential in the field of electromagnetic field protection, but it is particularly difficult to apply in the field of power cables $[9,10]$. 
1.4. Innovation Points. In this paper, through the transmission line model of the triaxial test system, the shielding performance of the mismatched cable can be calculated directly through the test, which not only realizes the rapid test of the shielding performance of the cable but also eliminates the possible error caused by the impedance matching network. In addition, this paper also studies the suppression effect of wiring and grounding methods on cable coupling, which expands the service conditions of cables and is conducive to the application of practical cables.

\section{Electromagnetic Field Shielding Method}

British scholar Maxwell summarized previous studies in 1864, put forward a complete electromagnetic wave theory, affirmed the existence of electromagnetic waves, and proposed that electromagnetic waves propagate forward at the speed of light [11]. The electromagnetic wave is one of the motion forms of the electromagnetic field. It propagates in the form of a wave by in-phase oscillation in space and vertical electric and magnetic fields. Its propagation direction is perpendicular to the plane formed by an electric field and magnetic field, which effectively transmits energy and momentum. Both show that changing the electric field will produce a magnetic field, and changing the magnetic field will produce an electric field [12]. From a scientific point of view, the electromagnetic wave is a kind of energy. Anything higher than absolute zero will release electromagnetic waves, and the higher the temperature, the shorter the wavelength of the electromagnetic wave, that is, the higher the frequency of the electromagnetic wave. The propagation and radiation of the electromagnetic wave will interfere with the operation of the power cable and its surrounding environment. Electromagnetic radiation hazards are mainly manifested in ignition caused by electromagnetic energy, combustion of volatile flammable substances, failure of safety-critical circuits, mistriggering of electric detonators, harmful biological effects on the human body, or gradual degradation when equipment, human body, ordnance, or fuel are exposed to dangerous electromagnetic radiation environment [13].

\subsection{Principle of Shielding Electromagnetic Field Materials.} When the electromagnetic wave propagates to the shielding surface because the constituent elements of the shielding layer include metal materials, part of the incident wave energy is shielded and reflected. If the electromagnetic wave propagates inside the material, part of the energy is absorbed and consumed, and only a small amount of energy can be transmitted to the air through the material [14]. Electric and magnetic fields can excite each other through motion, so there is a certain coordinate system conversion relationship between electric and magnetic fields. Therefore, ordinary electric and magnetic fields can be regarded as a manifestation of electromagnetic fields in a specific coordinate system and unified into electromagnetic field tensors Express. The principle is shown in Figure 1.
2.1.1. Reflection of Electromagnetic Wave. If the characteristic impedance of electromagnetic wave in medium 1 is $Z 1$, then medium 2 is $Z 2$, the magnetic field strength is $Q$, and the incident electric field strength of electromagnetic wave is $R 0$; then, the electric field strength and magnetic field strength of the reflected wave reflected back to medium 1 on interface 1 are, respectively,

$$
\begin{gathered}
R_{r 1}=\frac{Z_{1}-Z_{2}}{Z_{1}+Z_{2}} R_{0}, \\
Q_{r 1}=\frac{Z_{2}-Z_{1}}{Z_{1}+Z_{2}} Q_{0} .
\end{gathered}
$$

Since the radiation source is relatively fixed, the amount of radiation each time is also relatively fixed, and the amount of radiation reflected each time is also completely absorbed. After the electromagnetic wave passes through interface 1 and enters medium 2, the electric field and magnetic field intensity of the electromagnetic wave are, respectively,

$$
\begin{gathered}
R_{1}=R_{0}-R_{r 1}=\frac{2 Z_{2}}{Z_{1}+Z_{2}} R_{0}, \\
Q_{1}=Q_{0}-Q_{r 1}=\frac{2 Z_{1}}{Z_{1}+Z_{2}} Q_{0} .
\end{gathered}
$$

Generally, the existing shielding materials have a certain thickness, so after penetrating into such materials, the electromagnetic wave will be reflected many times, part of the energy will be absorbed, and the rest will be transmitted to the air [15].

2.1.2. Electromagnetic Wave Absorption. When the electromagnetic wave enters the absorbing medium, the electromagnetic field intensity decays exponentially with the depth of entering the medium, which is due to the induced eddy current generated when the electromagnetic wave enters the medium [16]. $H$ is the thickness of the material, $D$ is the distance from the electromagnetic source, and the electric and magnetic field strength formulas of the rest are as follows:

$$
\begin{aligned}
& R_{1}=R_{0} * e^{-h / d}, \\
& Q_{1}=Q_{0} * e^{-h / d} .
\end{aligned}
$$

According to Einstein's photon theory. The electromagnetic wave energy calculation formula is $E=\operatorname{nh} \gamma=\mathrm{nhc} / \lambda$, where $n$ is the number of photons, $h$ is the Planck constant, $\gamma$ is the frequency, and $\lambda$ is the wavelength of electromagnetic waves in a vacuum. Figure 2 is the schematic diagram.

2.1.3. Multiple Reflection of Electromagnetic Wave. When the electromagnetic wave enters the shielding material, the shielding material has a certain thickness and will refract many times inside the material. Assuming that the electromagnetic wave intensity of the first reflection is Qr1 and Qr1, respectively, the electromagnetic wave intensity of the 
second refraction is $\mathrm{Rr} 2$ and Qr2, respectively, and the electromagnetic wave intensity of the second refraction back medium 1 is Rr3 and Qr3 [17], according to the reflection mechanism of electromagnetic wave, the following formula can be known.

$$
\begin{aligned}
& R_{r 1}=\frac{2 Z_{2}}{Z_{1}+Z_{2}} R_{0}, \\
& Q_{r 1}=\frac{2 Z_{1}}{Z_{1}+Z_{2}} Q_{0} .
\end{aligned}
$$

Thus, it can be deduced that

$$
\begin{aligned}
& R_{r 2}=\frac{Z_{1}-Z_{2}}{Z_{1}+Z_{2}}, \\
& R_{r 1}=\frac{\left(Z_{2}-Z_{1}\right) 2 Z_{2}}{\left(Z_{1}+Z_{2}\right)^{2}} R_{0}, \\
& R_{r 2}=\frac{Z_{1}-Z_{2}}{Z_{1}+Z_{2}}, \\
& R_{r 1}=\frac{\left(Z_{2}-Z_{1}\right) 2 Z_{2}}{\left(Z_{1}+Z_{2}\right)^{2}} R_{0} .
\end{aligned}
$$

Then, you can get

$$
\begin{gathered}
R_{r 3}=R_{r 1}-R_{r 2}=\frac{4 Z_{1} Z_{2}}{\left(Z_{1}+Z_{2}\right)^{2}} R_{0}, \\
Q_{r 3}=Q_{r 1}-Q_{r 2}=\frac{4 Z_{1} Z_{2}}{\left(Z_{1}+Z_{2}\right)^{2}} Q_{0} .
\end{gathered}
$$

The method of measuring the resistivity of the insulator is the same as that of the conductor resistivity. The volume resistivity of the insulator can be obtained by dividing the total resistance by the volume of the insulator, and the surface resistivity of the insulator can be obtained by dividing the total resistance by the surface area of the insulator. Generally speaking, the electromagnetic shielding material contains a certain amount of metal fibers, and its surrounding environment is basically air (generally insulated). Therefore, it can be seen that the reflection of electromagnetic radiation is the most basic way to shield electromagnetic waves [18]. For the magnetic field component, when the electromagnetic wave is reflected at the second interface, its magnetic field intensity is almost twice that of the initial incident electromagnetic wave; that is, the magnetic field intensity will become stronger after reflection, so the attenuation of the magnetic field is mainly through internal absorption [19].

2.2. Suppression Principle of Electromagnetic Coupling by Shielding Layer. The magnetic field coupling model mainly obtains the magnetic field coupling voltage by analyzing the mutual inductance between parallel cables. Passive magnetic field shielding refers to the use of a shielding layer in a disturbed cable to achieve the purpose of magnetic shielding.
Three-dimensional coil modeling that crosses vertically, different coil types, and excitation settings are different; the impedance parameters of the coil will also be different. Magnetic field shielding is the same as electric field shielding, and the method of shielding interfering cable and disturbed cable can be adopted [20]. The equivalent circuit diagram is shown in Figure 3.

From the above figure, it can be seen from Faraday's law that the current $I 1$ in the interference cable is induced by magnetic field coupling in the disturbed cable reference ground plane circuit to obtain the induced voltage $V 12$, which is

$$
V_{12}=\mathrm{jwM}_{12} I_{1} \text {. }
$$

At the same time, the magnetic field coupling induction of current $I 1$ in the shielding layer reference ground plane circuit obtains the induced voltage v1s, which is

$$
V_{1 s}=\mathrm{jwM}_{1 s} I_{1} \text {. }
$$

When the shielding layer is not grounded or only one end is grounded, the induced voltage still exists in the shielding layer. However, since there is no circuit loop and no current passes through the shielding layer, the loop magnetic field will not be generated, which will affect the spatial magnetic field distribution generated by the interfering cable, and the mutual inductance between the interfering cable and the disturbed cable will remain unchanged [21]. As the magnetic flux changes, an induced current will be generated in the closed coil, and the induced current will generate a magnetic field. Therefore, the ungrounded or single-ended grounding of the shielding layer has no effect on the interference voltage V12 on the disturbed cable. When both ends of the shielding layer are grounded, the shielding layer and the reference ground plane form a circuit loop, and the induced current flowing in the shielding layer is as follows:

$$
I_{s}=\frac{\mathrm{jwM}_{1 s} I_{1}}{Z_{s}+\mathrm{jwP}_{s}} .
$$

In the above formula, PS is the self-inductance of the ground plane loop of the shielding layer and ZS is the impedance of the shielding layer [22]. Due to the secondary effect, the induced current in the shielding layer will couple the induced voltage v2s in the disturbed cable reference ground plane loop and the coupling voltage $\mathrm{v} 2 \mathrm{~s}$ is as follows:

$$
V_{2 s}=\mathrm{jwM}_{2 s} I_{s}
$$

As can be seen from the passive shielding equivalent circuit diagram, the induced voltage in the disturbed cable is

$$
V_{2}=V_{12}-V_{2 s}=\mathrm{jwM}_{12} I_{1}-\mathrm{jwM}_{2 s} I_{s} \text {. }
$$

According to the mutual inductance theory,

$$
\begin{aligned}
& M_{12}=M_{1 s}, \\
& M_{2 s}=L_{s} .
\end{aligned}
$$

By solving the above formula, 


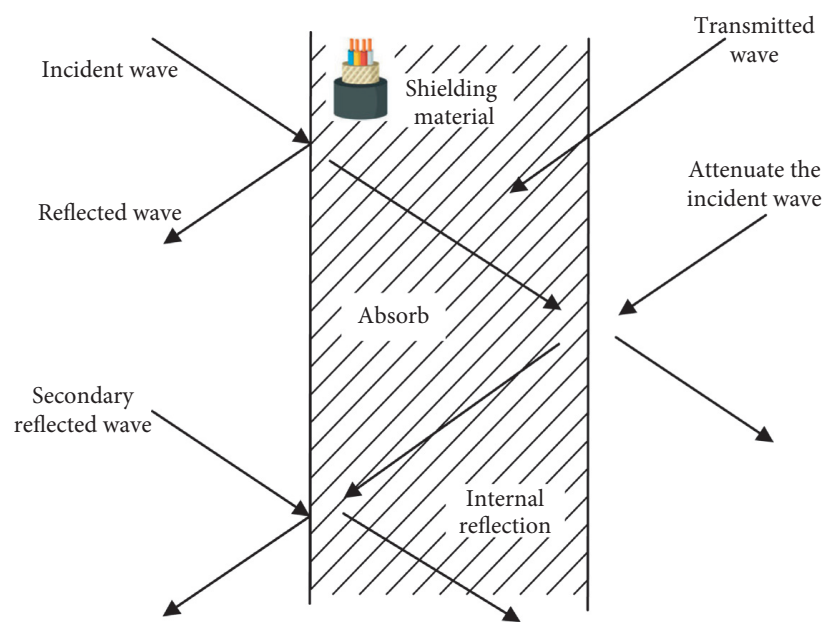

FIgURE 1: Attenuation principle of shielding material to electromagnetic wave.

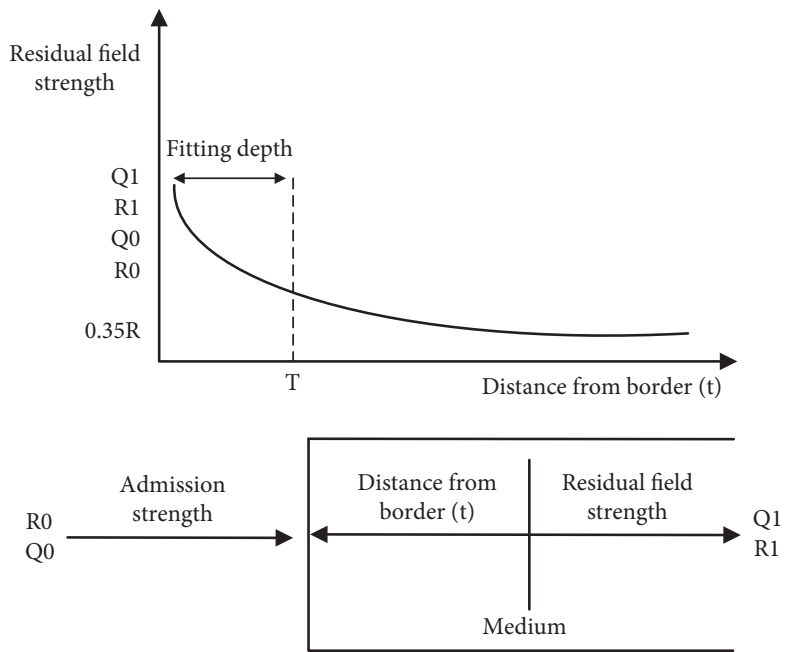

FIgURE 2: The electromagnetic wave decays exponentially through the shield.

$$
V_{2}=\frac{j w M_{12} Z_{s}}{Z_{s}+j w L_{s}} I_{1} .
$$

The above formula is rewritten as

$$
V_{2}=\mathrm{jwM}_{12} I_{1}\left(\frac{Z_{s} / L_{s}}{j w+Z_{s} / L_{s}}\right) \text {. }
$$

When frequency $w<Z_{s} / L_{s}$, then $\left(Z_{s} / L_{s} / \mathrm{jw}+Z_{s} / L_{s}\right) \approx 1$. At this time, the induced voltage $V 2$ is

$$
V_{2} \approx \mathrm{jwM}_{12} I_{1} \text {. }
$$

It can be seen from the comparative equations (8) and (16) that $V 2$ and $V 12$ are almost equal, indicating that even the double terminal grounding treatment of the shielding layer cannot provide magnetic field shielding at low frequency [23]. According to equation (7), the cut-off frequency of the shielding layer is $W_{1}=Z_{s} / L_{s}$. When $w>W 1$, that is, when the frequency of the interference magnetic field is much higher than the cut-off frequency of the shielding layer, the induced voltage $V 2$ is as follows:

$$
V_{2} \approx M_{12} I_{1} \frac{Z_{s}}{L_{s}}
$$

Equation (15) is plotted in combination with equations (16) and (17), as shown in Figure 4.

It is not difficult to see from the above figure that when $w>W C$, the induced voltage no longer increases with the change of frequency but remains unchanged. At low frequency, the coupling induced voltage in the disturbed cable with and without the shielding layer is equal, indicating that the shielding layer has no shielding effect at this time. In the above case, in fact, the magnetic induction coupling between the power cable and signal cable is mainly low-frequency magnetic field coupling, and its working frequency is mostly $50 \mathrm{~Hz}$. However, the cut-off frequency of nonmagnetic shielding layer in the signal cable is $0.6-7.0 \mathrm{kHz}$, and the working frequency of the interference magnetic field is much lower than that of the nonmagnetic shielding layer [24]. The effect of the shielding layer is not mainly due to the reflection and absorption of the electric field and magnetic field by the metal body itself, but due to the grounding of the shielding 


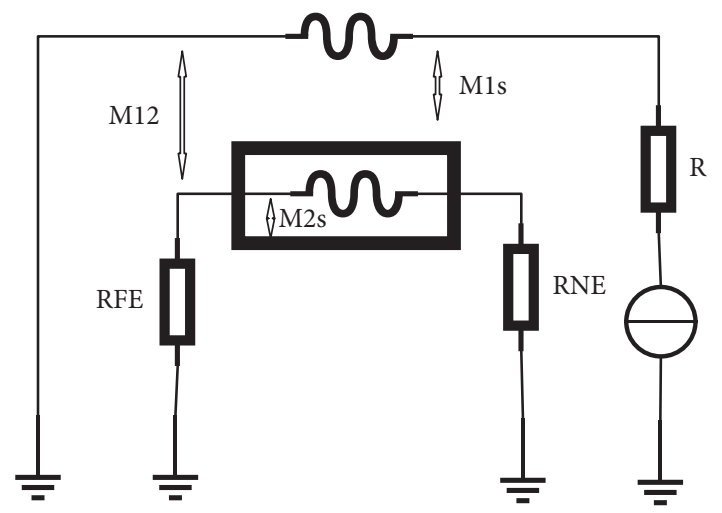

FIGURE 3: Schematic diagram of magnetic field passive shielding.

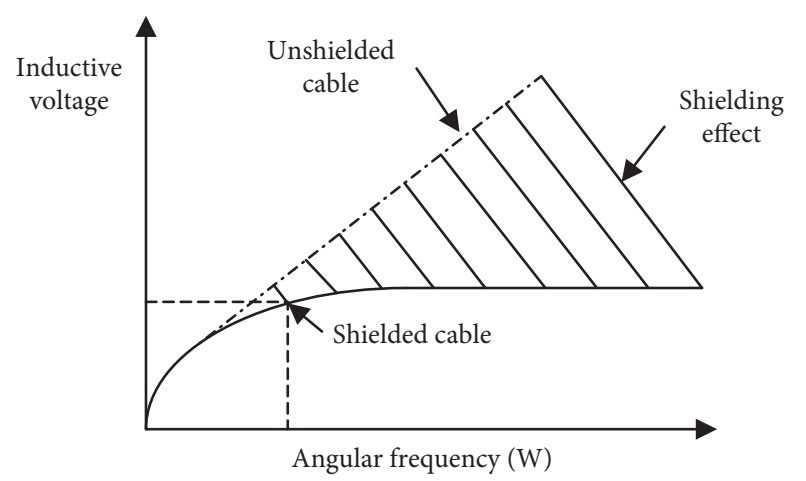

FIgURE 4: Relationship between induced voltage and frequency.

layer. Different forms of grounding will directly affect the shielding effect. At this time, the nonmagnetic shielding layer has no shielding effect on the coupling interference of the low-frequency magnetic field. When shielded by the high-frequency magnetic field, the interference coupling frequency is much higher than the cut-off frequency of the nonmagnetic shielding layer. When the nonmagnetic shielding layer is well grounded and both ends are grounded, it has good active shielding and passive shielding suppression effects on magnetic field coupling [25].

\section{Electromagnetic Field Detection Analysis and Shielding Experiment of Power Cable}

3.1. Triaxial Magnetic Field Impedance Measurement. In this experiment, syv 1 and syv 4 coaxial power cables are selected as the test objects. In the actual design, by optimizing the finger period of the interdigital transducer, surface acoustic waves of different wavelengths are excited, and the resonant frequency of the surface acoustic wave device is adjusted. Data Transfer Process. Their characteristic impedances are $50 \Omega$ and $75 \Omega$, respectively. They are coaxial cables often used in EMC experiments. The specific parameters are shown in Table 1.

If the load is only different in power, the core wire diameter of the cable is different, and everything else can be the same. If the type of load is also different, the type of cable may also be different. It can be seen from the table that except for different inner conductor diameters, the structural dimensions of the two coaxial cables are the same, and the braiding pitch, number of braided strands, and number of braided wires of each strand of the braided layers of the two coaxial cables are also the same. According to the transfer impedance theory of braided coaxial cables in Section 2, the transfer impedance of the shielding layers of the two coaxial cables is the same. Therefore, these two types of cables are selected to compare the effects of different matching states on cable shielding performance [26]. The experimental test equipment includes a laboratory-made triaxial device, vector network analyzer, commercial impedance matching network, and connecting cable [27].

The specific test steps are as follows: calibrate the two ports of the connecting cable to eliminate the error caused by the transmission loss of the connecting cable; pass the prepared cable under test through the triaxial clamp; connect both ends of the cable shielding layer to the semicircular copper block; short-circuit the cable shielding layer and the triaxial outer loop copper pipe; connect the output end of the network analyzer to the inner ring of the triaxial system. The input terminal is connected to its external circuit [28]. The return loss S11 and transmission loss S21 of the three coaxial systems are measured by the vector network analyzer. The tested cables are divided into five groups to test the transfer impedance of cables under different matching states. See Table 2 for details.

The resistance of a conductor is proportional to the length and inversely proportional to the size of the crosssectional area. The ratio of resistance to cable length used in this article is between 0 and 0.4 . Test results of syv1 coaxial power cable are as follows.

It can be seen from Figure 5 that the theoretical calculation results are in good agreement with the actual trend compared with the experimental test results, and the test results are higher than the calculation results. At low frequency, the transmission impedance of the cable is approximately equal to the DC impedance of the shielding layer, and the theoretical value is about $10 \mathrm{~m} \Omega / \mathrm{m}$ at $3 \mathrm{MHz}$. However, for the triaxial test system, the inner ring and the outer ring are connected by transfer impedance. The test results include the impedance of the copper tube in the outer ring. In addition, the dynamic range of the test system is small, resulting in the test results being slightly larger than the theoretical value. In the test, the metal tube outside the triaxial device will radiate energy to the outside, which will also make the test result too large [29]. On the other hand, the triaxial device calculates the transfer impedance of the cable through the formula by testing the S21 parameters of the tested cable. Therefore, the use of secondary parameters may lead to errors in the theoretical value. The transfer impedance measured at low frequency is the DC impedance of the cable, which is about $10 \mathrm{~m} \Omega / \mathrm{m}$. With the increase of frequency, the scattering impedance becomes the main form of transfer impedance. At $10 \mathrm{MHz}$, the transfer impedance is about $50 \mathrm{~m} \Omega / \mathrm{m}$. At high frequency, the hole inductance and braided inductance are the main factors. The electromagnetic energy is coupled to the cable core through the holes on the cable shielding layer, and the transfer impedance 
TABLE 1: Structural dimensions of syv1 and syv4 coaxial power cables.

\begin{tabular}{lcccccc}
\hline \multirow{2}{*}{ Specification } & \multicolumn{2}{c}{ Inner conductor } & Insulation outer diameter $(\mathrm{mm})$ & \multicolumn{2}{c}{ Shield } & Outer diameter \\
& Material & Diameter $(\mathrm{mm})$ & Material & Diameter $(\mathrm{mm})$ & $(\mathrm{mm})$ \\
\hline SYV1 & Soft copper wire & 0.5 & 5 & Soft copper wire & $0.14 \sim 0.16$ \\
SYV4 & Soft copper wire & 0.75 & 5 & Soft copper wire & $0.14 \sim 0.16$ & 6.8 \\
\hline
\end{tabular}

TABLE 2: Grouping of tested cables.

\begin{tabular}{|c|c|c|c|c|}
\hline $\begin{array}{l}\text { Serial } \\
\text { number }\end{array}$ & $\begin{array}{c}\text { Test } \\
\text { model }\end{array}$ & $\begin{array}{l}\text { Cable characteristic impedance } \\
(\Omega)\end{array}$ & $\begin{array}{c}\text { Cable terminal resistance } \\
(\Omega)\end{array}$ & Matching status of signal source and cable \\
\hline Test one & SYV1 & 50 & 50 & Match \\
\hline Test two & SYV4 & 75 & 75 & $\begin{array}{c}\text { No impedance matching network, single-ended } \\
\text { mismatch }\end{array}$ \\
\hline Test three & SYV4 & 75 & 75 & $\begin{array}{c}\text { Connect to 50-75 impedance matching network, } \\
\text { matching }\end{array}$ \\
\hline Test four & SYV4 & 75 & 50 & $\begin{array}{l}\text { No impedance matching network, double-ended } \\
\text { mismatch }\end{array}$ \\
\hline Test five & SYV4 & 75 & 50 & $\begin{array}{c}\text { Connect to 50-75 impedance matching network, } \\
\text { matching }\end{array}$ \\
\hline
\end{tabular}

increases with the increase of frequency [30]. The impedance fluctuation value in this article is too small, so the error of the test impedance will not affect the safety of the cable.

The measured S21 parameters of syv4 cable terminal with $75 \Omega$ and $50 \Omega$ matching load are shown in the figure and compared with the test results of syvl cable. The measured S21 parameters when the terminals do not match are shown in Figure 6.

It can be seen from the figure that at $75 \Omega$, the attenuation caused by the mismatch between the start end of the tested cable and the end face of the signal source makes the measured S21 parameters less than the value in the matching state in the whole frequency band. The single-end mismatch only affects the attenuation and will not fluctuate. At $50 \Omega$, the S21 measured by the mismatched cable is more consistent with the results measured by the matched cable at low frequency, but the difference becomes larger and larger with the increase of frequency; that is, the impact of mismatch becomes larger and larger. The attenuation is zero at low frequency and becomes larger and larger with the increase of frequency.

In the low-voltage power distribution system, the current carrying capacity of the wires and cables needs to be selectively matched with the protective appliances. According to different situations, the mismatch between the wire and the protective appliances can be roughly divided into two types. The transfer impedance of the tested cable is calculated from the S21 parameters obtained from the test and compared with the test results of the syv1 cable, as shown in Figure 7.

It can be seen from the figure that the difference between the test results of S21 and the matching cable measured when the terminals are not matched increases with the increase of the frequency, so the test results of the mismatched state and the matched state in the figure are almost the same at low frequencies. But as the frequency increases, the difference between the two increases. Due to the mismatch of the tested cable terminals, although the impedance matching network is connected between the signal source and the tested cable during the syv 4 cable test, the results also differ greatly from the test results of the syvl cable matching state with the increase of frequency.

In conclusion, when measuring the transfer impedance of mismatched cables by the three coaxial methods, the calculation result of the measurement formula when the tested cable is terminated with matching load is better than that when the terminal is connected with $50 \Omega$ load. Therefore, when testing unmatched cables, the more accurate test results can be obtained by using the test method of terminal connection matching load and calculation with the measurement formula.

\subsection{Suppression of Electromagnetic Interference by Magnetic} Materials. Magnetic materials are important functional materials with a wide range of applications and varieties. According to the application types, they can be divided into soft magnetic, permanent magnetic, and other materials. For the electromagnetic field shielding solution, the experimental work of this paper mainly includes, by analyzing the active and passive shielding under different coupling interference conditions, the suppression effect of shielding on the electric field and the magnetic field is obtained [31]. The power cable adopts magnetic material as the shielding layer, which can suppress the low-frequency magnetic field, which is verified by simulation. Using magnetic material as the shielding layer of signal cable, the time domain value is obtained through experimental measurement, the frequency domain value is obtained through FFT transformation, the structure is shown in Figure 8, and the suppression effect of the low-frequency magnetic field is analyzed.

The main objective of this experiment is to develop electromagnetic field shielding of underground variable frequency power cable, so as to meet the safety and reliability of the cable system. Experiments are designed to analyze the suppression effect of magnetic materials on active low- 


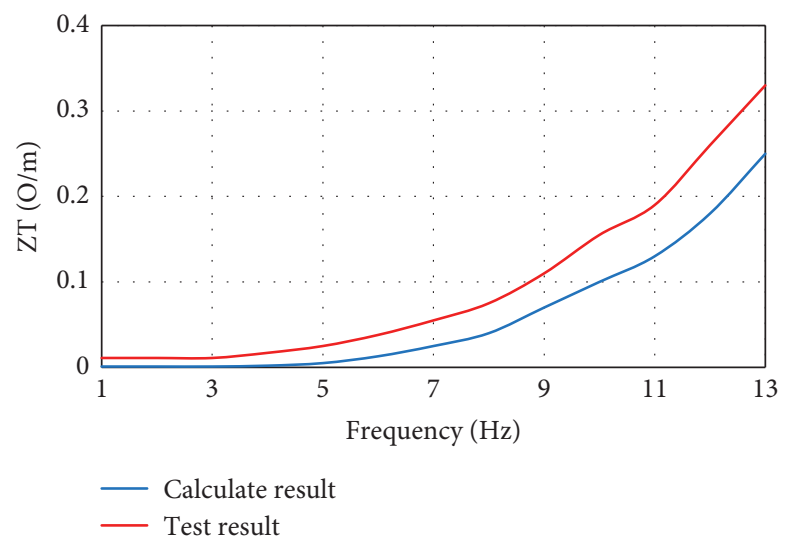

FIGURE 5: Transfer impedance of syv1 power cable.
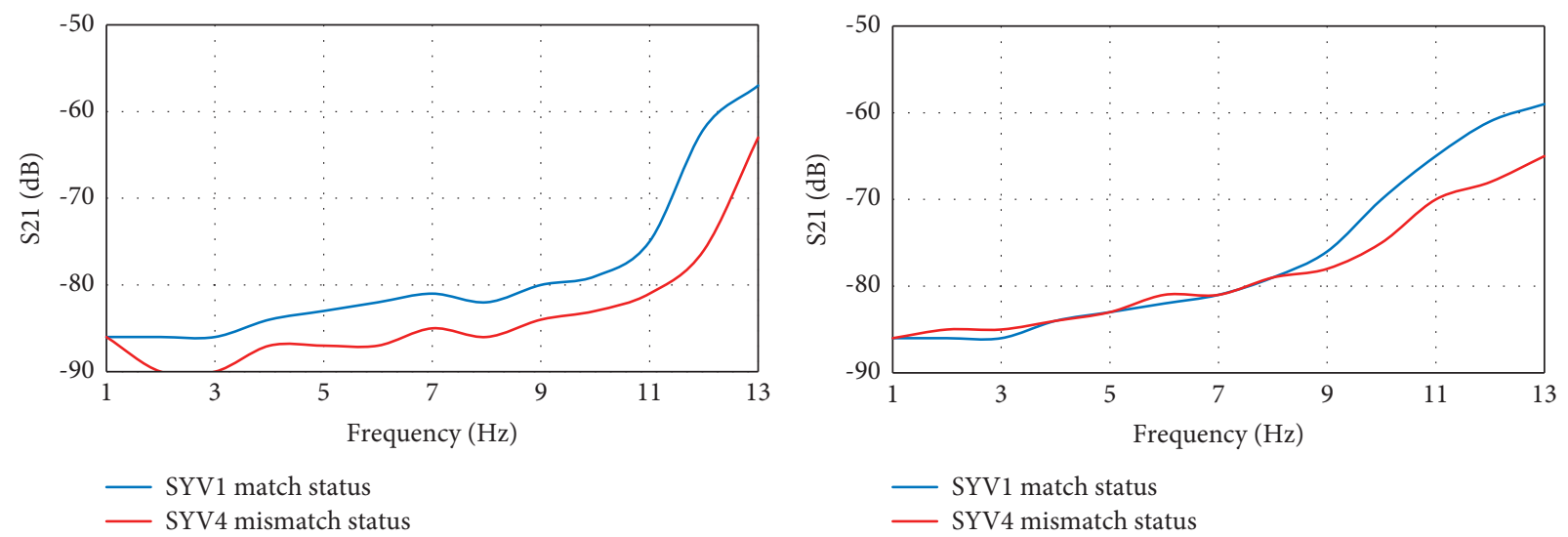

FIGURE 6: S21 parameters measured when the power cable is connected to $75 \Omega$ and $50 \Omega$ loads.

frequency magnetic field coupling in power cables, and the experimental results are verified by simulation calculation.

We arrange the two power cables in close parallel, analyze the magnetic field distribution around the loop cable, and select the horizontal $x$-axis and vertical $Y$-axis of the cable for magnetic field measurement respectively, and the measurement steps are consistent with the measurement of a single circuit power cable. The Gauss meter probe is close to the cable with the $x$-axis and $y$-axis as the measurement starting position, and the measurement direction of the probe is perpendicular to the magnetic field direction. After that, the probe moves outward, the distance between measuring points is $1 \mathrm{~cm}$, and we measure and record the data. We keep the loop current at $70 \mathrm{~A}$ and measure it according to the above steps. During the test, the external ambient magnetic field is $-0.273 \mathrm{GS}$, and the actual magnetic field value is the measured value minus the ambient value [32]. The test results are shown in Table 3.

It can be seen from Table 3 that when the loop cable does not use the shielding layer, the attenuation law of the magnetic field strength along the $x$-axis and $y$-axis around the cable is the same as that of the single-channel cable. This article uses Ampere's law to obtain the predicted value of the magnetic induction intensity of the cable, and the prediction and actual comparison can be used to obtain the magnitude of the error by the prediction method proposed in this article. Both attenuate with the increase of distance and increase with the distance. The degree of attenuation is reduced. And the magnitude of the magnetic field along the $x$-axis at the same distance is smaller than the magnitude of the magnetic field along the $y$-axis.

Data processing is performed on the measurement results, and the processing results are shown in Figure 9.

It can be seen from the above figure that before the shielding material is used, the magnetic field distribution of the $x$-axis and $y$-axis is still nonlinear, and the attenuation trend is consistent with the formula. At the same distance, the magnetic field intensity of the $y$-axis is greater than that of the $x$-axis, which is also consistent with the fact that the magnetic field intensity generated by the ring cable at the center is reversed in the $x$-axis direction and the $y$-axis is in the same phase. Therefore, the magnetic field intensity in the $y$-axis direction is equal to the sum of the $y$ components of the magnetic field intensity of the ring cable in the $y$-axis direction. The magnetic field intensity in the $x$-axis direction is equal to the difference of the $\mathrm{Y}$ component of the magnetic field intensity in the $x$-axis direction of the ring cable. As can be seen in Figure 9, when the loop cable is wrapped with 

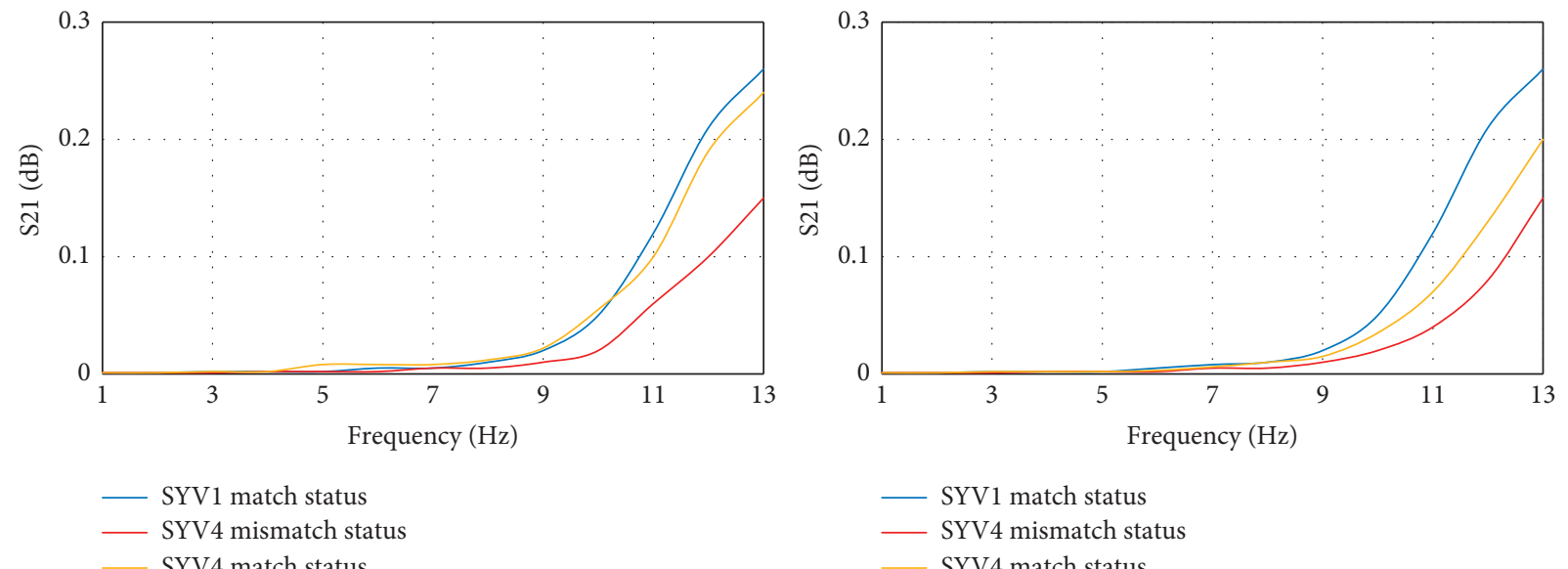

- SYV1 match status
SYV4 mismatch status

Figure 7: Power cable connected to $75 \Omega$ and $50 \Omega$ resistance transfer impedance.

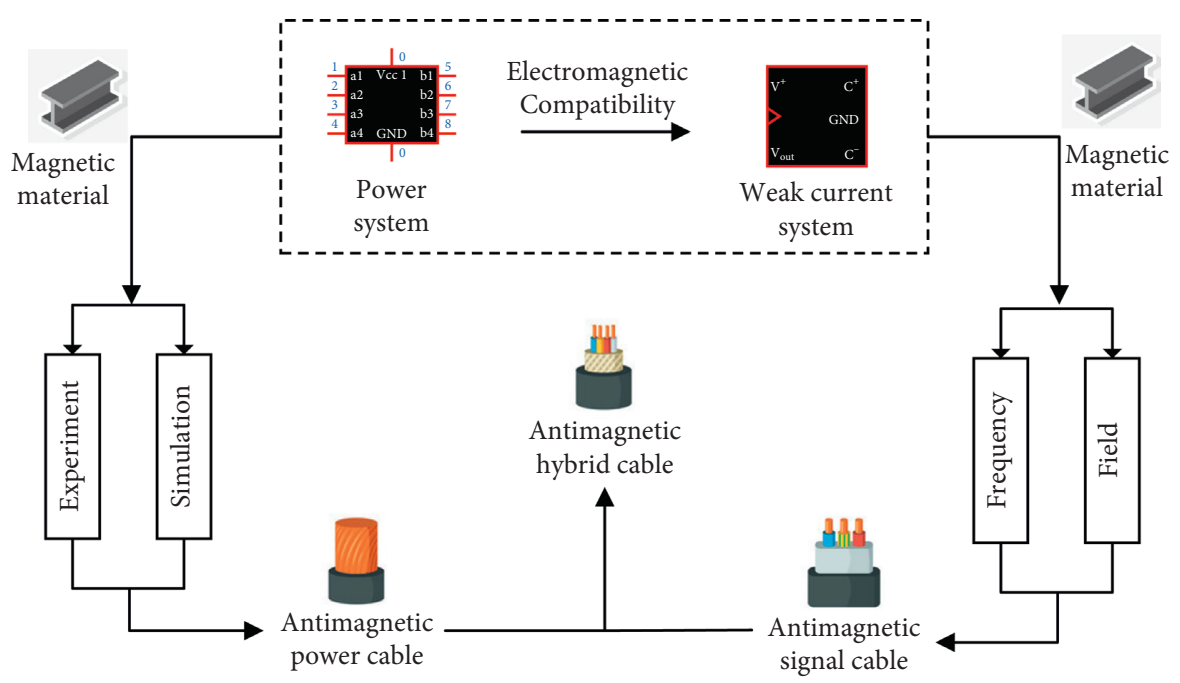

Figure 8: Shielding scheme.

magnetic materials, the magnetic field in both the $x$-axis and $Y$-axis directions is obviously suppressed, and the measured magnetic field strength is almost zero. By calculating the magnetic field efficiency along the $x$-axis and $y$-axis, it can be found that after using the magnetic material as the shielding layer, the shielding efficiency at the initial measurement point is the largest, which is $32.8 \mathrm{~dB}$ and $26.4 \mathrm{~dB}$, respectively, and then the shielding efficiency decreases with the increase of distance.

The above experimental measurement results are explained below. The magnetic fields on both sides of the multi-interface suddenly change according to a certain law, and this sudden change relationship is called the boundary value problem or boundary condition of the magnetic field. Data visualization is represented in the four quadrants. The magnetic field generated by the cable will enter the magnetic material, and the direction of the magnetic field generated by the cable in the magnetic material can be obtained from the boundary conditions of the magnetic medium interface, as shown in Figure 10.
The figure above shows the normal boundary condition of magnetic flux density and tangential boundary condition of magnetic field strength. At the interface of permeability and magnetic medium, the unit vector of normal points from the third quadrant to the first quadrant, and the magnetic flux density and magnetic field strength on both sides of the interface are Q1 and Q2, respectively. The results show that magnetic materials have an obvious inhibitory effect on the electromagnetic field. There is no problem for the cable covering material to absorb radiation in a short time, and it will cause leakage if it takes a long time, so it needs to be replaced in time.

\subsection{Performance Test of Electromagnetic Shielding Materials.} In this paper, three commonly used electromagnetic shielding materials are selected, which are (1) metal fiber radiation protection material, (2) high fiber radiation proof fabric, and (3) silver fiber antiradiation materials. See Table 4 for the specifications of metal-plated antiradiation materials. 
TABLE 3: Magnetic induction intensity of loop cable during operation.

\begin{tabular}{lcccc}
\hline Distance $(\mathrm{CM})$ & $X$-measurement $(\mathrm{GS})$ & $X$-actual value $(\mathrm{GS})$ & $Y$-measurement $(\mathrm{GS})$ & $Y$-actual value $(\mathrm{GS})$ \\
\hline 0 & 4.697 & 5.77 & 15.999 & 15.872 \\
1 & 1.587 & 2.36 & 4.829 & 2.201 \\
2 & 0.794 & 1.067 & 1.539 & 2.074 \\
3 & 0.797 & 0.67 & 1.474 & 1.312 \\
4 & -0.336 & 0.237 & 0.381 & 1.347 \\
5 & -0.06 & 0.713 & 0.952 & 1.454 \\
6 & 0.668 & 0.841 & 0.671 & 0.744 \\
7 & -0.083 & 0.19 & 0.726 & 1.399 \\
8 & 0.169 & -0.058 & 0.793 & 0.866 \\
9 & -0.659 & -0.186 & 0.362 & 1.235 \\
\hline
\end{tabular}
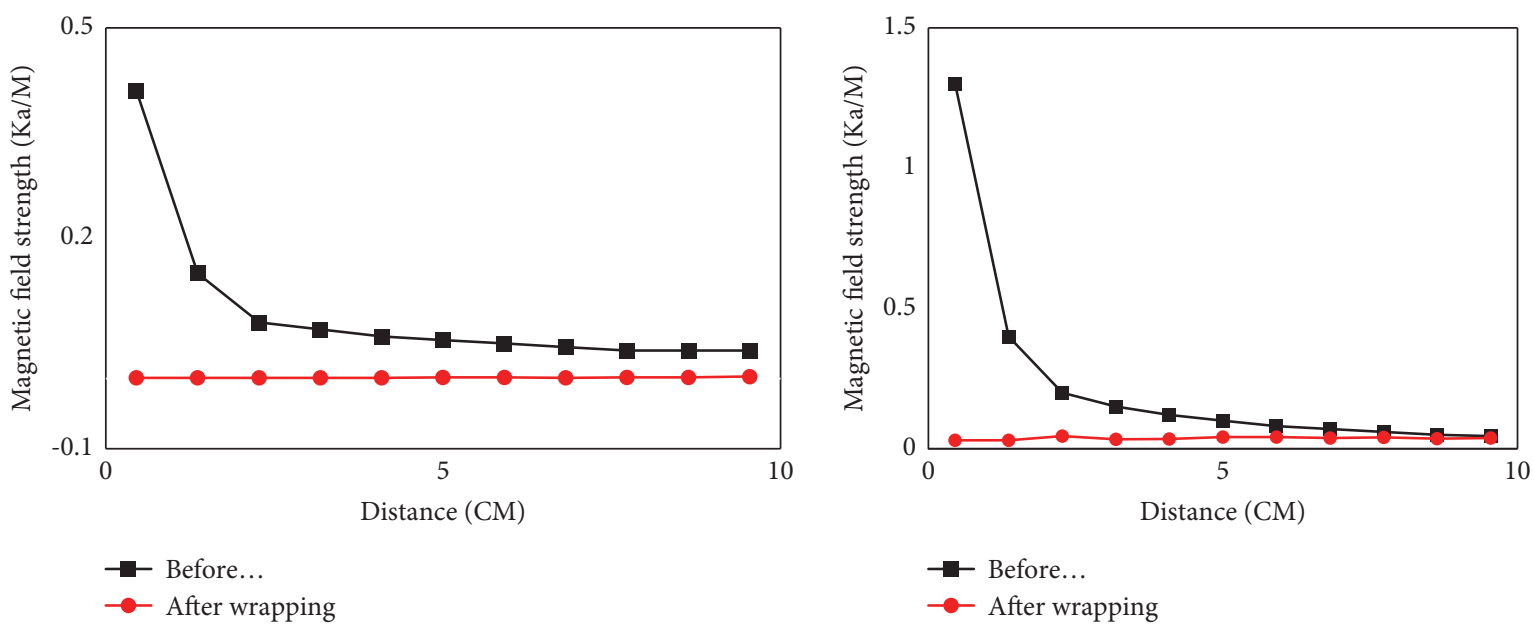

FIgURE 9: Variation of the magnetic field along $x$-axis and $y$-axis.

This experiment uses the shielded room method. Power cables, especially ultrahigh voltage transmission lines, should be kept away from densely populated areas such as houses, schools, and sports fields, and the distance between the human body and the cables should be no less than $75 \mathrm{~cm}$, and it is best to install shielding devices. We use a combination of the spectrum analyzer, shielded room, and transmitting antenna, including wireless LAN standard antenna, horn antenna, mobile phone standard antenna, coaxial cable, and connector, test in a shielded room with a temperature of $25^{\circ} \mathrm{C}$ and a humidity of $65 \%$, and keep the distance between the antenna and the transmitting antenna at $3 \mathrm{~m}$. Although the result of the experiment is that the material with the highest density and thickness has achieved advantages, the fundamental reason for the shielding effectiveness is the material, so there is no need to uniformly test the density and thickness of the material. The shielding effectiveness test results of different materials at different frequencies are shown in Figure 11.

According to the hygienic standard of environmental electromagnetic waves, the minimum shielding efficiency that can protect the human body is $25 \mathrm{~dB}$. It can be seen from the figure that the shielding efficiency of the three materials is greater than $25 \mathrm{~dB}$, so as to meet the requirements of protecting the human body. When the same fabric is tested, its shielding efficiency will decrease slightly with the increase of radiation source frequency. When the radiation sources are the same, the shielding effectiveness of different materials is also different. Among them, material 2 has the best shielding efficiency, followed by material 1 , and material 3 has the worst shielding efficiency. The main reasons for the above differences are, as follows. First, the composition of the materials is different. The metal fiber of the materials is copper-nickel alloy. Generally, the conductivity of the alloy is worse than that of pure metal, so some electromagnetic waves can be better reflected back to the air. Second, different structures of materials lead to different compactness of materials, of which materials 1 and 3 are plain and materials 2 are twill. Generally speaking, twill materials have fewer warp and weft interleaving times, which reduces the gap between warp and weft, and the yarns can be closely arranged. Therefore, the density of the materials is higher and thicker. Therefore, the shielding efficiency of the two materials is the highest, with an average efficiency of $76.4 \mathrm{~dB}$.

\section{Discussion}

Although this thesis derives the measurement formula for mismatched cable shielding performance, there are still many problems that need to be solved and improved. (1) 


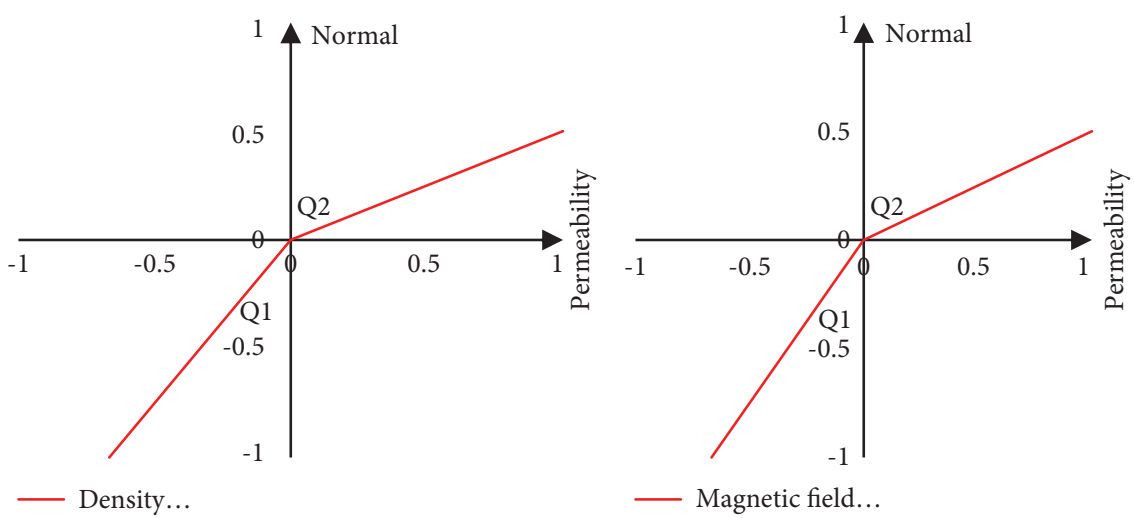

Figure 10: Boundary conditions of the magnetic medium interface.

TABLE 4: Specifications of electromagnetic shielding materials.

\begin{tabular}{llcccc}
\hline \multirow{2}{*}{ Number } & \multirow{2}{*}{ Name } & \multirow{2}{*}{ Fabric structure } & \multicolumn{2}{c}{ Density } & \multirow{2}{*}{ Mass per square meter (g) } \\
\cline { 3 - 5 } & & & Meridional & Latitudinal & 56.4 \\
2 & Metal-plated antiradiation fabric & Plain weave & 9 & 9 & 184.5 \\
3 & High fiber radiation proof fabric & Vertical grain & 27 & 27 & 81.2 \\
\hline
\end{tabular}

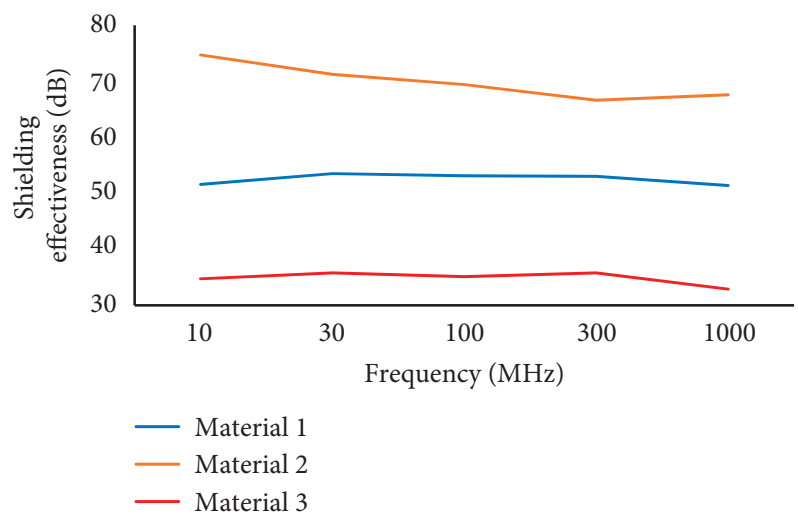

FIGURE 11: Shielding effectiveness test results of different materials.

This article only uses $50 \mathrm{ohms}$ and $75 \mathrm{ohms}$ braided shielded cables as examples to verify the measurement formula. In actual underground applications, cables of other specifications such as multicore cables, shielded twisted pairs, and twisted pairs are also used. It is widely used, so follow-up work needs to test and verify other specifications of cables. (2) Magnetic materials have a good suppression effect on low-frequency magnetic fields, but the permeability of magnetic materials decreases with the increase of frequency, and it is easy to increase the distortion of harmonics in the interference frequency under magnetic saturation. (3) When selecting shielding materials, the number of selected samples is relatively small, and it may be possible to try to recombine multiple materials to obtain a new multilayer shielding material. In future research, more practical materials and combinations can be selected.

\section{Conclusions}

The main conclusion of this paper is divided into two parts: one is the rapid detection of the electromagnetic field of underground variable frequency power cable; the other is the shielding of the electromagnetic field. Based on the multiconductor transmission line theory and the solution of multiconductor transmission line equation, according to the influence of cable pairing conditions on the cable shielding performance test, the transmission line model of the triaxial test system is established, and the measurement formula of unmatched cable shielding performance is deduced. The effectiveness of the formula is verified by experiments, and the error is no more than $8 \mathrm{~dB}$. Then, the electromagnetic interference suppression experiment of magnetic materials shows that the electromagnetic shielding performance of 
signal cable with appropriate magnetic materials as shielding layer can reach $20 \mathrm{~dB}$. Then, through the electromagnetic shielding performance test of three electromagnetic shielding materials, it is concluded that the shielding efficiency of metal fiber materials is the highest, with an average of $76.4 \mathrm{~dB}$.

\section{Data Availability}

The data that support the findings of this study are available from the corresponding author upon reasonable request.

\section{Conflicts of Interest}

The authors declare no potential conflicts of interest with respect to the research, authorship, and/or publication of this article.

\section{Acknowledgments}

This work was supported by Dynamic Monitoring and Risk Early Warning Technology System for Site Groundwater contamination (National Key R\&D Projects, 2019YFC1804804).

\section{References}

[1] C. Li, H. J. Yang, F. Sun, J. M. Cioffi, and L. Yang, "Multiuser overhearing for cooperative two-way multiantenna relays," IEEE Transactions on Vehicular Technology, vol. 65, no. 5, pp. 3796-3802, 2016.

[2] W. Li, V. Vaziri, S. S. Aphale, S. Dong, and M. Wiercigroch, "Dynamics and frequency and voltage control of downhole oil pumping system," Mechanical Systems and Signal Processing, vol. 139, no. May, pp. 106562.1-106562.24, 2020.

[3] J. Tian, L. Wei, and T. Zhang, "Dynamic research and experimental analysis of a new downhole drilling tool," Arabian Journal for Science and Engineering, vol. 44, no. 12, pp. 10231-10244, 2019.

[4] G. R. Samuel, "Kick detection using downhole accelerometer data," Journal of Energy Resources Technology, vol. 142, no. 8, pp. 1-13, 2020.

[5] S. C. Rajpoot, C. Pandey, P. S. Rajpoot, S. K. Singhai, and P. K. Sethy, "A dynamic-SUGPDS model for faults detection and isolation of underground power cable based on detection and isolation algorithm and smart sensors," Journal of Electrical Engineering \& Technology, vol. 16, no. 4, pp. 1799-1819, 2021.

[6] S. Famakin and C. Kim, "Modeling for underground cable water tree growth dynamics," Journal of Power and Energy Engineering, vol. 07, no. 12, pp. 51-65, 2019.

[7] M. K. Hadi, "Utilization of the finite element method for the calculation and examination of underground power cable ampacity," International Journal of Applied Power Engineering, vol. 8, no. 3, pp. 257-264, 2019.

[8] G. Lovat, P. Burghignoli, R. Araneo, E. Stracqualursi, and S. Celozzi, "Analytical evaluation of the low-frequency magnetic shielding of thin planar magnetic and conductive screens," IEEE Transactions on Electromagnetic Compatibility, vol. 63, no. 99, pp. 1-5, 2020.

[9] X. Ding, Yu Wang, R. Xu, Q. Qi, W. Wang, and D. Yu, "Layered cotton/rGO/NiWP fabric prepared by electroless plating for excellent electromagnetic shielding performance," Cellulose, vol. 26, no. 13-14, pp. 8209-8223, 2019.

[10] P. Wang, T. Yao, Z. Li, W. Wei, Q. Xie, and W. Duan, "Huilong Han, A superhydrophobic/electrothermal synergistically anti-icing strategy based on graphene composite," Composites Science and Technology, vol. 198, Article ID 108307, 2020.

[11] J. Li, K. Ren, H. D. Chen, H. L. Jiang, C. Wang, and T. Huang, "Characteristic analysis of downhole pressure signal while drilling under drillstring washout conditions," Materials Science Forum, vol. 944, no. 2, pp. 1040-1049, 2019.

[12] X.-x. Gao, X.-x. Pan, and G.-a. Zhu, "An analysis method for time-, frequency-, and energy-domain characteristics of downhole microseismic signals and its application," Shock and Vibration, vol. 2021, no. 2, pp. 1-12, 2021.

[13] A. G. Shcherbinin and R. P. Lukoyanov, "Numerical studies of the electric field of a power cable with sector strands and impregnated paper-plastic insulation for a voltage of $20 \mathrm{kV}$," Russian Electrical Engineering, vol. 91, no. 11, pp. 698-702, 2020.

[14] O. Kropotin, "Mathematical model of XLPE insulated cable power line with underground installation," Przeglad Elektrotechniczny, vol. 1, no. 6, pp. 79-82, 2019.

[15] M. Bhujanga, S. Surya, A. Sai, D. Prasad, T. Y. Satheesha, and K. N. Nagesh, "Underground cable fault using robot," World Journal of Nano Science and Engineering, vol. 4, no. 4, pp. 300-309, 2019.

[16] N. Murugan, J. S. Senthil Kumar, T. Thandapani, S. Jaganathan, and N. Ameer, "Underground cable fault detection using internet of things (IoT)," Journal of Computational and Theoretical Nanoscience, vol. 17, no. 8, pp. 3684-3688, 2020.

[17] Q. Li, C. Zhou, H. Wang, and T. Zhi, "Influence of redistribution circuit current in an underground cable trench on cable service life," Gaodianya Jishu/High Voltage Engineering, vol. 45, no. 6, pp. 1576-1583, 2019.

[18] R. Markowska, "Exposure of underground cable intrusion detection system to transient disturbances caused by nearby lightning strikes," Elektronika ir Elektrotechnika, vol. 26, no. 4, pp. 11-17, 2020.

[19] B. Akbal, "MSSB to prevent cable termination faults for long high voltage underground cable lines," Elektronika ir Elektrotechnika, vol. 25, no. 6, pp. 8-14, 2019.

[20] S.-K. Kang, M.-H. Park, Y.-H. Kim, N.-W. Kim, and I.-Y. Seo, "Development of anomaly-detection system for the underground cable tunnel using autoencoder," The Transactions of the Korean Institute of Electrical Engineers, vol. 69P, no. 2, pp. 69-75, 2020.

[21] T. Bragatto, M. Cresta, F. M. Gatta, A. Geri, M. Maccioni, and M. Paulucci, "A 3-D nonlinear thermal circuit model of underground MV power cables and their joints," Electric Power Systems Research, vol. 173, no. AUG, pp. 112-121, 2019.

[22] C. E. Mueller, S. I. Keil, and C. Bauer, "Underground cables vs. overhead lines: quasi-experimental evidence for the effects on public risk expectations, attitudes, and protest behavior," Energy Policy, vol. 125, no. FEB, pp. 456-466, 2019.

[23] H. Shabani and B. Vahidi, "A probabilistic approach for optimal power cable ampacity computation by considering uncertainty of parameters and economic constraints," International Journal of Electrical Power \& Energy Systems, vol. 106, no. MAR, pp. 432-443, 2019.

[24] X. Peng, L. Wang, J. Zhang, J. Chen, and B. Dai, “The field shielding effect of mountain on the lightning electromagnetic 
field," Journal of Electromagnetic Analysis and Applications, vol. 12 , no. 2, pp. 15-28, 2020.

[25] Z. Su, W. Lyu, L. Chen et al., "Shielding effect of surrounding buildings on the lightning-generated vertical electric field at the top of a tall building," IEEE Transactions on Electromagnetic Compatibility, vol. 61, no. 1, pp. 174-182, 2019.

[26] I. Kolcunová, J. Zbojovsk, M. Pavlík et al., "Shielding effectiveness of electromagnetic field by specially developed shielding coating," Acta Physica Polonica A, vol. 137, no. 5, pp. 711-713, 2020.

[27] N. A. Litvinova and I. S. Kyrie, "Study of the shielding ability of mesh metal materials from the electromagnetic field of industrial frequency power lines in urban environments," Ecology and Industry of Russia, vol. 25, no. 2, pp. 28-33, 2021.

[28] Y. I. Gabrousenko, A. G. Holubnychyi, Z. Hu, A. G. Taranenko, and F. J. Yanovsky, "Estimation of electromagnetic shielding efficiency," Telecommunications and Radio Engineering, vol. 78, no. 2, pp. 181-189, 2019.

[29] Y. Li, D. Su, S. Cui, and Li Weimin, "Feasibility analysis of the installation of a guided-wave HEMP simulator in an electromagnetic shielding room," Applied Computational Electromagnetics Society Journal, vol. 34, no. 6, pp. 970-976, 2019.

[30] G. Lovat, P. Burghignoli, R. Araneo, and S. Celozzi, "Magnetic shielding of planar metallic screens: a new analytical closedform solution," IEEE Transactions on Electromagnetic Compatibility, vol. 62, no. 99, pp. 1-9, 2019.

[31] B. Elhadi, H. Abdechafik, G. Bachir, and B. Aissa, "Influence of plastic deformation of copper on the behavior of electromagnetic shielding," Annales de Chimie Science des Matériaux, vol. 43, no. 3, pp. 135-140, 2019.

[32] H. Shrivastav, T. Enomoto, S. Seto, K. Araki, and C. Hwang, "Near-field scanning-based shielding effectiveness extraction for board-level shielding cans," IEEE Transactions on Electromagnetic Compatibility, vol. 63, no. 99, pp. 1-11, 2021. 